\section{Nicolas Poujol} Charles Sultan

\title{
Action moléculaire des androgènes et relation structure-fonction du récepteur des androgènes
}

Les androgènes influencent la différenciation, la croissance et le développement d'une grande variété de cellules aussi bien chez l'homme que chez la femme. Ils exercent leurs effets en se liant au récepteur des androgènes, l'un des membres de la superfamille des récepteurs nucléaires, capables de moduler la transcription de gènes cibles. La fixation du ligand par le récepteur des androgènes cytoplasmique, lui même lié à diverses protéines (les protéines de choc thermiques ou hsp pour heat shock proteins), va déclencher une série de modifications (départ des $h s p$, hyperphosphorylations). Le récepteur des androgènes subit alors une translocation jusqu'au noyau, où il va reconnaître ses éléments de réponse hormonaux et recruter le complexe activateur de transcription. Ces deux dernières années ont été marquées par des avancées significatives dans la compréhension de l'action cellulaire et moléculaire des androgènes. Le rôle des acteurs impliqués dans l'activation transcriptionnelle du récepteur des androgènes est de mieux en mieux compris. L'analyse tridimensionnelle des domaines de liaison de l'ADN et de l'hormone laisse entrevoir de nombreuses applications en physiopathologie et en pharmacologie.

\footnotetext{
ADRESSES

N. Poujol : Inserm U. 439, Pathologie moléculaire des récepteurs nucléaires, 70 , rue de Navacelles, 34090 Montpellier; Laboratoire d'hormonologie du développement et de la reproduction, Hôpital Lapeyronie, Montpellier. Adresse actuelle: Inserm U. 414, Centre de biochimie structurale, 29, rue de Navacelles, 34090 Montpellier. C. Sultan Inserm U. 439, Pathologie moléculaire des récepteurs nucléaires, 70 , rue de Navacelles, 34090 Montpellier; Laboratoire d'hormonologie du développement et de la reproduction, Hôpital Lapeyronie, Montpellier; Endocrinologie et gynécologie pédiatriques, Pédiatrie 1, Hôpital §.-de-Villeneuve, Montpellier, France.
}

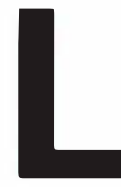

es androgènes sont responsables de la différenciation, du développement, et du maintien du phénotype masculin. ('es effets sont exercés par le biais d'un récepteur nucléaire qui, après liaison de la testostérone (T) ou de la dihydrotestostérone
(I)HT), se fixe sur des séquences d'ADN spécifiques situées en amont de gènes cibles, et active (voire réprime) la transcription de ces derniers. Parmi les gènes cibles, on peut citer l' $A B P$ dans le testicule de rat, la probasine, le ISA clans la prostate, qui sont considérés plus comme des 
marqueurs que comme des effecteurs de l'action des androgènes.

Toute altération de l'une des étapes de la transmission du message par les androgènes peut conduire au développement d'anomalies aussi diverses que le syndrome d'insensibilité aux androgènes [1], des processus néoplasiques (cancer de la prostate et cancer du sein chez. l'homme), une atteinte neurodégénérative (maladie de Kennedy) ou une stérilité isolée. Ces maladies représentent de véritables «modèles expérimentaux » qui contribuent à mieux cerner le mécanisme d'action des androgènes dans leurs cellules cibles.

Le syndrome d'insensibilité aux androgènes représente plus de la moitié des cas de pseudo-hermaphrodisme masculin [2]. Son expression clinique est très variable, pouvant aller d'un phénotype féminin complet à un phénotype masculin avec ambiguïté sexuelle (pour revue, voir [3]). La génétique moléculaire permet aujourd'hui d'affirmer le diagnostic de l'anomalie par l'identification de mutations dans le gène codant pour le récepteur des androgènes.

En réalité, les anomalies moléculaires des androgènes ne pourront être cernées qu'après avoir établi une carte détaillée des relations structure-fonction du récepteur des androgènes, précisé ses multiples partenaires et élucidé le mode d'action cellulaire et moléculaire des androgènes.

\section{Le récepteur des androgènes}

Le récepteur des androgènes (AR) appartient à la superfamille des récepteurs nucléaires qui comprend: - les récepteurs d'hormones stéroïdes, androgènes ( $\mathrm{AR} A$ et isof orme B), glucocorticoïdes $(G R \alpha, \beta)$, minéralocorticoïdes (MR), progestérone $\left(\mathrm{PR}_{\left.\mathrm{A},{ }_{B}\right)}\right)$ et œestrogènes $(\mathrm{ER} \alpha, \beta)$;

- les récepteurs de différents ligands hydrophobes tels que les hormones thyroïdiennes (TR $\alpha, \beta)$, l'acide rétinoïque ( $\operatorname{RAR} \alpha, \beta$, $\gamma$ et $\operatorname{RXR} \alpha, \beta, \gamma)$.

- les récepteurs activant la prolif ération des péroxysomes (PPAR);

- de nombreux récepteurs orphelins pour lesquels aucun ligand spécifique n'est identifié (pour revue, voir [4]).

L'AR est organisé, tout comme selon une structure modulaire avec des domaines fonctionnels interchangeables (figure 1):

- le domaine amino-terminal impliqué dans l'activation de la transcription (AF I) et participant à la spécificité d'action tissulaire et cellulaire;

- le domaine central de liaison à l'ADN (DBD pour DNA binding domain), fortement conservé au sein de la superfamille. Il participe aussi au processus de dimérisation;

- le domaine charnière abritant un signal de localisation nucléaire (NL1, pour nuclear localization) ;

- le domaine carboxy-terminal responsable de la liaison de l'hormone (LBD, pour ligand binding domain) mais possédant également une surface d'interaction pour les protéines de choc thermique (hsp), une fonction de transactivation dépendant de la liaison du ligand (AF2), une surface impliquée dans les processus de dimérisation et un signal de localisation nucléaire hormono-dépendant (NL2).

Le gène du AR est situé sur le chromosome $X$, en position qll-ql2 et s'étend sur environ $90 \mathrm{~kb}$ (figure 1). Le cadre ouvert de lecture comprend 8 exons et s'étend sur $2,7 \mathrm{~kb}$. Plusieurs isoformes d'ARNm ont été décrites chez. l'homme.
Exprimé dans la plupart des tissus, le AR présente un taux d'expression variant d'un facteur 2 à 3 . En outre, de profondes différences d'expression peuvent être observées [5] au sein d'une même cellule, en fonction du développement ou de l'âge. Le promoteur du AR ne possède pas de boîtes TATA, ni CAAT. En revanche, il possède un élément de réponse à l'AMPc, des sites potentiels de liaison pour une vingtaine de facteurs transcriptionnels tels que SRY, SF1, le AR lui-même, ER ou encore NFKB. Deux sites de liaison du AR ont aussi été décrits dans l'exon 5 [6]. Enfin, nous avons observé une régulation biphasique de l'expression de l'ARNm du récepteur des androgènes par les androgènes [7]

\section{Mode d'action du récepteur des androgènes}

\section{La liaison de l'hormone}

L'existence de divers domaines structuraux au sein des récepteurs stéroïdiens a été révélée par des expériences de protéolyse ménagée. La résolution des structures tridimensionnelles des LBD de plusieurs récepteurs de la superfamille en

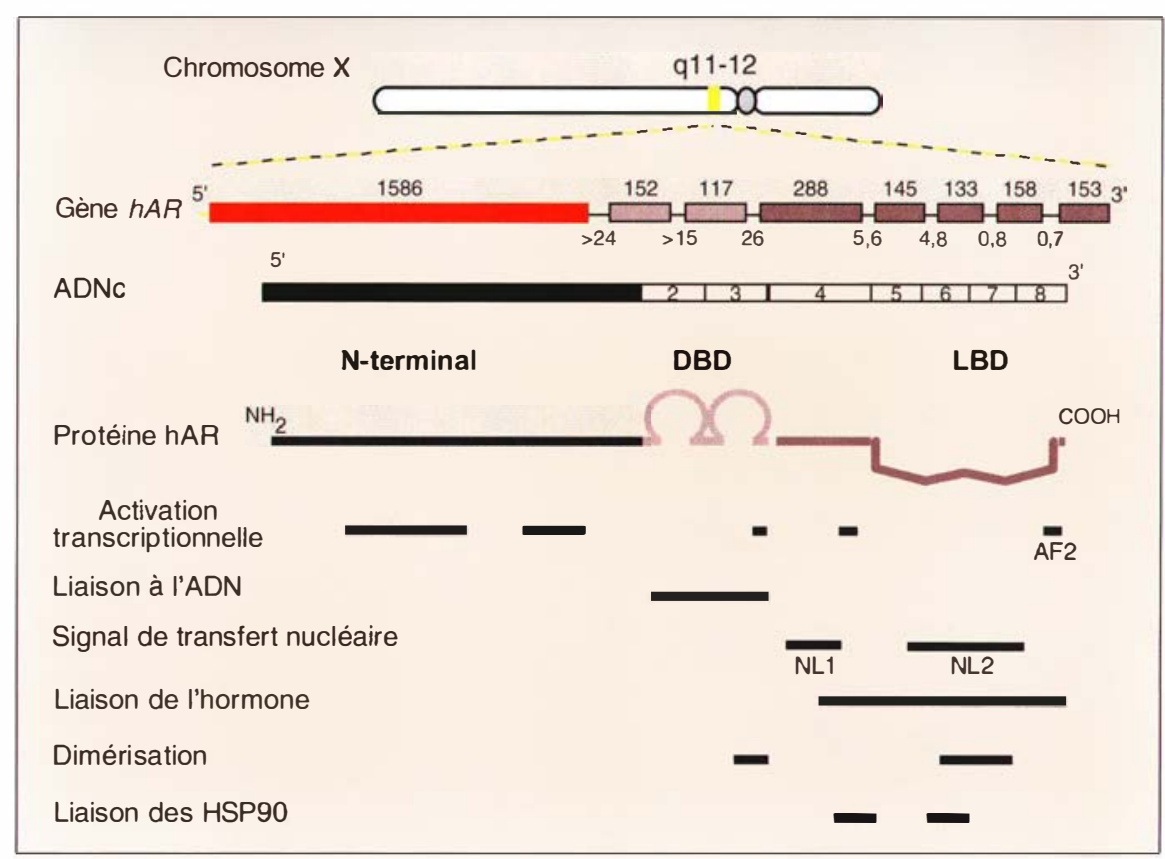

Figure 1. Organisation génique et principaux domaines fonctionnels du récepteur des androgènes. 
l'absence de ligand (apo-), en présence d'agoniste (holo-) ou d'antagoniste ainsi que la prédiction d'une architecture générale commune à tous les LBD ont par la suite considérablement enrichi nos connaissances en matière d'activation des récepteurs nucléaires.

\section{Structure canonique du domaine de liaison de l'hormone.}

A ce jour, les structures cristallines de 6 IBD des récepteurs nucléaires suivants ont été résolues, $\mathrm{RXR} \alpha$ apodimèrique, RAR $\gamma$ et TR $\alpha$ holo-monomérique, ER $\alpha$ holo-dimérique formant un complexe avec divers ligands, PPAR $\gamma$ apo- et holo-dimćrique et $\mathrm{PR}$ holo-dimérique [8-10].

Les structures de $\operatorname{RXR} \alpha$ et $\operatorname{RAR} \gamma$ ont toutes deux révélé un repliement original appelé "sandwich d'hélices $\alpha$ ». L'éloignement phylogénique de ces deux récepteurs a suggéré la conservation d'une architecture générale commune [11] au sein de la superfamille, qui fut ultérieurement retrouvée dans des récepteurs aussi divers que le TR, l'ER, le PR et le PPAR. L'ensemble de ces données nous a permis de réaliser in silico, par homologie avec les structures du RAR $\gamma$, de l'ER $\alpha$ et du PR, un modèle tridimensionnel du domaine de liaison de l'hormone du récepteur des androgènes, pour lequel il n'existe pas de structures cristallines résolues à l'heure actuelle. Nous disposons ainsi d'un "guide" autorisant une approche de mutagenèse raisonnée, dont le but est de déterminer les modalités d'ancrage du ligand dans la poche, ou encore de préciser les résidus participant à des interactions protéine-protéine.

\section{Poche de liaison de l'hormone}

Tous ces LBD présentent donc une architecture comparable avec, pour le AR la présence de 11 hélices $\alpha$ ( $\mathrm{Hl}$ à H12) et d'un feuillet $\beta$ (S1/S2) (figure 2A). Les hélices $\mathrm{H} 4,5,6,8,9$ sont prises en sandwich entre $\mathrm{Hl}$ et 3 d'une part et $\mathrm{H} 7,10$ et 11 d'autre part.

La poche de liaison est particulièrement conservée, le ligand étant ancré au cour de la molécule. Les ligands sont reconnus grâce à 2 types de contacts: des liaisons hydrogène aux extrémités et des liaisons hydrophobes de type van der Waals pour $\mathrm{m} / \mathrm{s} n^{\circ} 6-7$, vol. 16, juin-juillet 20()() tout le corps du ligand. La poche est en effet tapissée de résidus dont le caractère hydrophobe est complémentaire du caractère apolaire des ligands, quclques rares résidus polaires assurant l'ancrage et la spécificité de reconnaissance. L'ancrage du cycle A des stéroïdes apparaît très conservé. Une approche de mutagenèse dirigée, guidée par un modèle tridimensionnel du LBD de hMR, a permis à Fagard et al. de préciser les modalités de reconnaissance des minéralocorticoïdes [12], que nous avons étendues aux androgènes, l'ancrage de la cétone en 3 étant réalisé par R752 et Q711 (figure 2B).

A l'autre extrémité du ligand, le mécanisme d'ancrage n'est pas aussi clairement mis en évidence par le cristal de PR. Dans le cas du AR, le résidu N705 semble particulièrement important, sa substitution par une alanine entraînant une perte drastique de liaison des androgènes, un important décalage de la courbe d'activation transcriptionnelle [13], les mêmes effets étant observés pour le résidu homologue de MR [12].

Le volume des poches de liaison des récepteurs stéroïdiens est bien supérieur à celui des ligands qu'elles doivent reconnaître $(341 \AA$ et $236 \AA$, respectivement, pour le $A R$ et la testostérone) ce qui peut s'expliquer par l'extrême rigidité des stéroïdes, qui n'autorise pas d'adaptation possible. Cette différence de volume réitère l'importance des contacts spécifiques et permet de comprendre que des molécules très diverses puissent être reconnues par ces récepteurs, mais bien souvent avec des affinités faibles ou sans réel capacité d'induction de transcription. Ce modèle est ainsi un outil de choix dans la définition de nouveaux ligands, agonistes ou antagonistes, une fois connus les points d'ancrages possibles au sein de la poche de liaison.

\section{Importance du positionnement de H12}

L'importance d'AF2 dans les phénomènes d'activation transcriptionnelle a été démontrée par mutagenèse dirigée, cela bien avant la résolution des structures tridimensionnelles. Or, la comparaison des structures apo- et holo- de $\operatorname{RAR} \gamma$ et $\operatorname{RXR} \alpha$ a révélé un positionnement différent de certains éléments structuraux, notamment de la dernière hélice $\alpha$, H12. Ceci a conduit Renaud et al. [14] à proposer un mécanisme d'activation commun à tous les récepteurs nucléaires: la fixation du ligand entraîne un repositionnement de H12 qui dans sa position finale, scelle ce demier dans la poche et crée avec la partie N-terminale de $\mathrm{H} 3$ une surface d'interaction qui autorise le recrutement de co-activateurs assurant la transduction du signal hormonal. Les bases structurales de cette interaction ont depuis été partiellement dévoilées $[9,10]$. Tout ligand induisant une position de $\mathrm{Hl} 2$ de type non holo-, et empêchant ainsi tout ou partie du recrutement de co-activateurs, va agir comme un antagoniste plus ou moins fort. On voit ainsi les applications possibles du modèle tridimensionnel pour la définition de nouveaux ligands.

\section{Application clinique}

Ce modèle in silico permet aussi de tenter d'interpréter rétrospectivement les répercussions structurales des nombreuses mutations naturelles du AR. Dans la figure 3, nous avons ainsi représenté par des couleurs différentes (rouge, insensibilité complète aux androgènes; bleu, insensibilité partielle aux androgènes, violet, cancer de prostate), toutes les substitutions rapportées dans la base de données sur le modèle tridimensionnel [15]. La répartition est assez homogène pour les 3 types d'anomalies, même si certains domaines sont plus fréquemment associés à des ICA, comme le cœur hydrophobe. Nous avons entrepris une étude systématique de ces altérations. Certaines sont aisément compréhensibles (perte de l'ancrage en 3-céto pour $\mathrm{R} 752 \mathrm{Q}$, perte d'un pont salin très conservé pour E681K), alors que d'autres amènent à s'interroger sur l'organisation structurale de la région dans laquelle elles se situent, notamment lorsqu'il s'agit de boucles.

\section{Activation et translocation nucléaire}

L'ancrage du ligand dans la poche, et les modifications de conformation induites, représentent les premières étapes de l'activation du récepteur des androgènes. Les étapes ultérieures visent à transformer ce récep- 


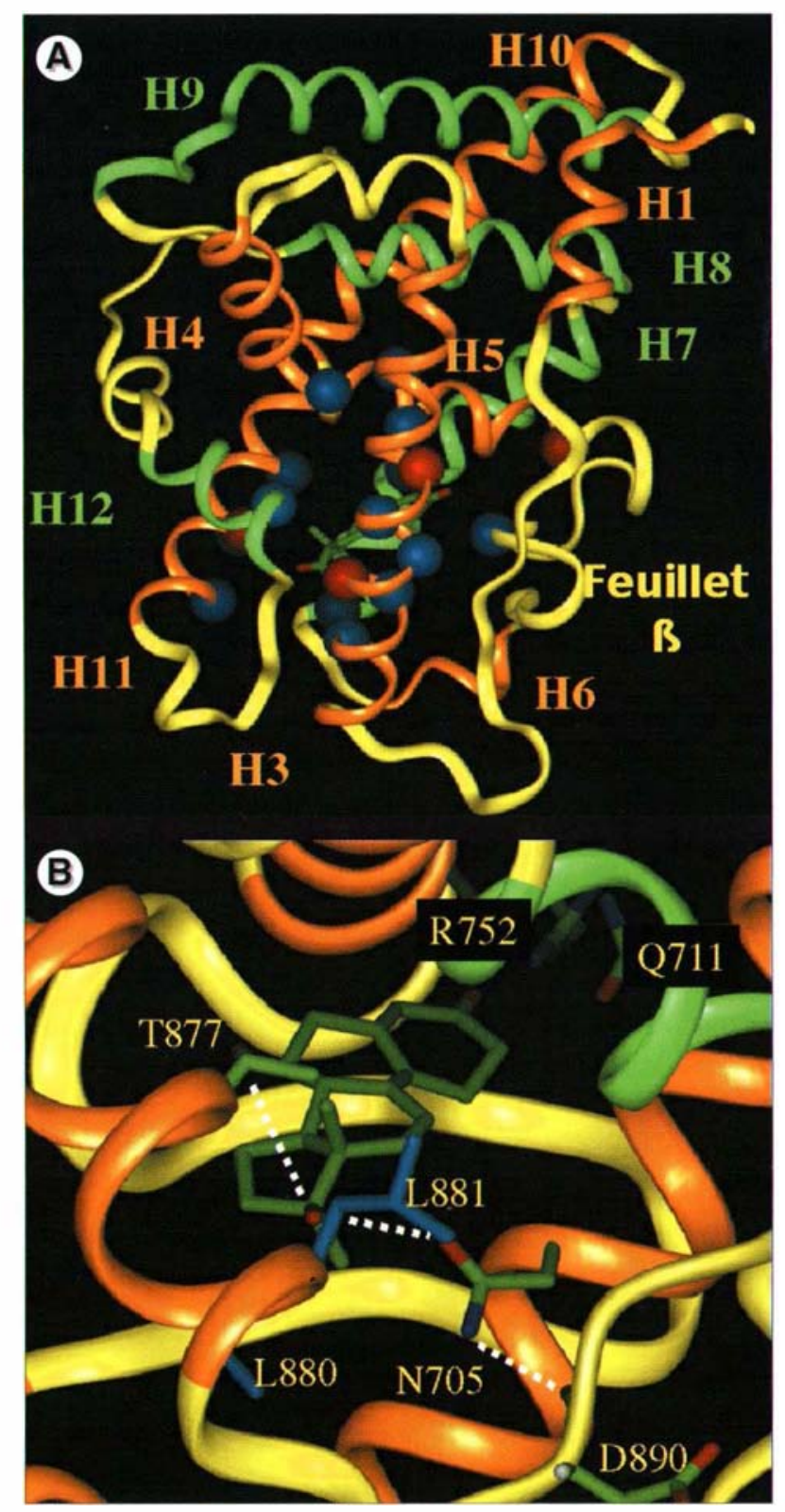

Figure 2. A. Vue de I'architecture générale de liaison de I'hormone du récepteur des androgènes en présence de R1881, modèle 30 réalisé à partir des coordonnées du complexe hPR-Progestérone. Les acides aminés à proximité immédiate du ligand $(4,5 \AA)$ sont représentés sous forme de sphères bleues pour les hydrophobes, rouges pour les polaires. B. Ancrage du R1881 au sein de la poche de liaison du récepteur des androgènes. Le code couleur conventionnel est appliqué $(C$ : vert $\mathrm{O}$ : rouge; $\mathrm{N}$ : bleu) à l'exception des résidus hydrophobes en bleu clair. L'ancrage du stéroïde est représenté en pointillé blanc.

teur cytoplasmique en l'absence d'hormone, formant un complexe avec des protéines de choc thermique (hsp), en facteur transcriptionnel nucléaire actif, donc capable de se lier à l'ADN et d'enclencher la transcription.

\section{Modifications induites}

par la liaison de l'agoniste

En l'absence de ligand, le AR est extrait des cellules sous la forme d'hétérocomplexes de hauts poids moléculaires $(25()-300 \mathrm{kDa})$ avant un coefficient de sédimentation de 8 à $10 \mathrm{~S}$

En présence de ligand, les protéines
$\mathrm{AR}$ sédimente à 4 à $5 \mathrm{~S}$ et acquiert la capacité de se lier à l'ADN. De nombreuses protéines constituant cet hétérocomplexe ont déjà été identifiées, telles que hsp90, hsp70 et FKBP52 [16].

Hsp 90 se lie au I.BD, et permet le maintien d'une conformation du AR apte à lier l'hormonc. Hsp 70, cytoplasmique, se lie très rapidement aux protéines en cours de synthèse et participe à l'assemblage d'oligomères. Un monomère d'hsp70 interagit directement avec le I.BD du AR, contrairement à FKBPs2, associée aux hsp90 avec l'AR, le PR, le GR, et l'ER dans l'hétérocomplexe.
La vitesse de dissociation des hsp correspond à la vitesse d'apparition d'un récepteur apte à lier l'ADN. I.e départ des hsp démasque le I.BD, les sites de dimérisation et le signal de localisation nucléaire. Les techniques de protéolyse ménagée ont permis de démontrer l'acquisition d'une structure plus compacte du récepteur et d'appréhender les modifications de conformation induites par la liaison d'agonistes ou d'antagonistes [17]. La fixation d'androgènes s'accompagne d'une augmentation de la phosphorylation du récepteur, selon un processus commun aux récepteurs stéroïdiens et à d'autres facteurs de transcription. A l'état inactif, le récepteur est déjà phosphorylé, mais cette hyperphosphorylation concerne de nouveaux sites [18]. Le rôle des phosphorylations dans l'activation des récepteurs nucléaires n'est pas clairement défini, mais plusieurs travaux tendent à démontrer son importance, même en l'absence de ligand, notamment dans l'activation de kinases dépendantes des cyclines et de MAP-kinases par des facteurs de croissance. Ainsi les phosphorylations de sérines spécifiques du domaine d'activation AFl de ERß [19] et de SFI [20] induites par les MAPK, aboutissent au recrutement de co-activateurs et à une activation transcriptionnelle. I.e contrôle des récepteurs nucléaires par ces phosphorylations reste tout de même mal connu, puisqu'en particulier leur spécificité d'action n'est pas clairement démontrée, leurs rôles physiologiques demeurant ainsi hypothétiques.

Le transfert nucléairc est sélectif, actif et nécessite un ou plusieurs NLS. La première étape de ce transfert est la liaison de la protéine aux pores nucléaires. Cette étape est indépendante de l'énergie, contrairement à la deuxième étape de translocation à travers les pores nucléaires. I'utilisation de deux inhibiteurs de synthèse d'ATP (désoxyglucose et acide de sodium) nous a permis de bloquer l'entrée dans le noyau d'une protéine de fusion (iFP-AR, démontrant clairement cette dépendance énergétique. Aujourd'hui, nous savons que le transport nucléaire est relayé par des récepteurs saturables qui effectuent un mouvement de va-et-vient entre le cytoplasme et le noyau (pour revue, voir [21]). Deux protéines ont été 


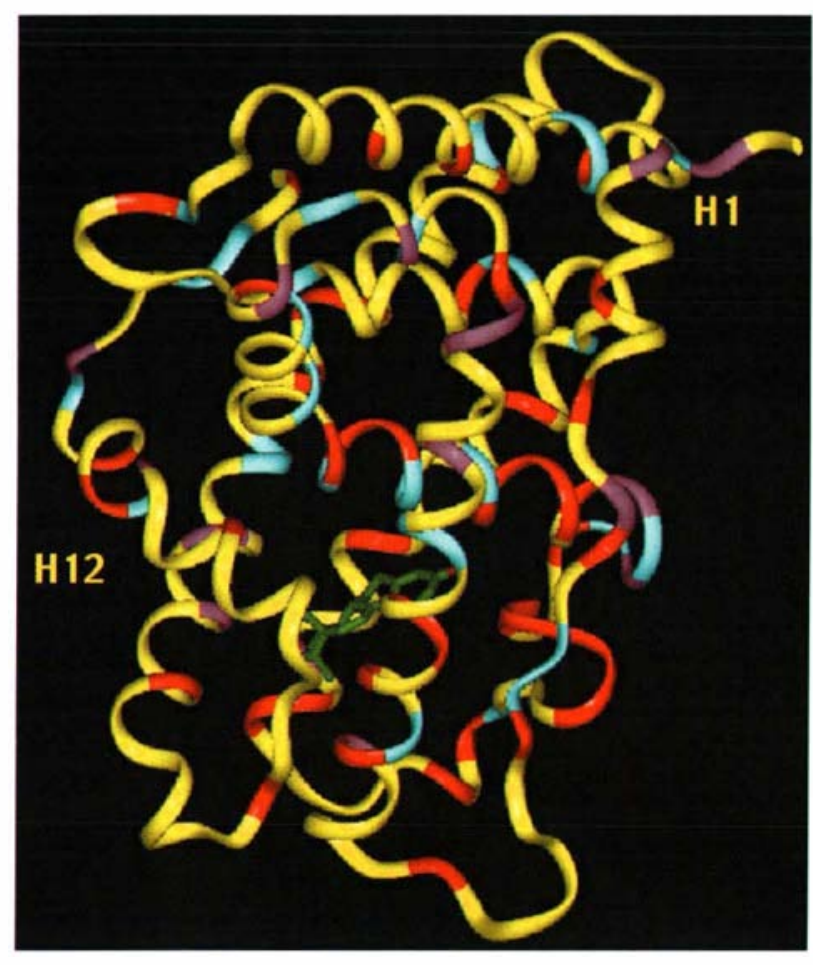

identifiées et leur gène respectif cloné par différents groupes, une protéine de $54 / 56 \mathrm{kDa}$, connue sous les noms d'importine- $\alpha$ ou karyophérine $\alpha$ et une protéine de $97 \mathrm{kl}$ )a, l’importine- $\beta$ ou karyophérine $\beta$.

\section{La liaison de l'ADN}

L'effet hormonal nécessite la liaison clu récepteur à de courtes séquences d'ADN mises en évidence principalement au niveau de la région promotrice de gènes cibles et appelées hormone responsive element (HRE). Afin d'identifier une séquence spécifique du AR, ou ARE, Roche et al. [22] ont utilisé un ensemble d'oligonucléotides et une protéine composée du scul DBD de AR. L'ARE alors sélectionné présente la séquence consensus $G G(A / T) A C A m n n T G T T C T$, très proche de la séquence consensus du GR. Tout ARE détecté coïncide avec un GRE, confortant la notion selon laquelle $\mathrm{GR} / \mathrm{AR} / \mathrm{PR} / \mathrm{MR}$ reconnaissent la même séquence, même si pour un certains nombre de gènes (mouse vas deferens protein, secretory component...) le AR semble plus efficace que le GR [23, 24]. Ceci soulève immédiatement le problème de la spécificité d'action dans les cellules exprimant les divers récepteurs.

$\mathrm{m} / \mathrm{s} n^{\circ} 6-7$, vol. 16, juin-juillet 20(0)
Figure 3. Modèle tridimensionnel du domaine de liaison de I'hormone du récepteur des androgènes. En rouge figurent les résidus dont les substitutions sont associées à des insensibilités complètes, en bleu celles associées à des insensibilités partielles et en violet celles associées à des cancers de la prostate.

ser les répercussions structurales de leur substitution [26]. Une approche en tout point identique pour les substitutions R607Q et R608k, identifiées dans des IPA et des cancers du sein chey l'homme, a mis en évidence une surface d'interaction potentielle au sommet du deuxième doigt de zinc [27].

\section{Dimérisation}

Il est communément admis que les récepteurs stéroïdiens se lient à l'ADN sous forme d'homodimères. Ceci scmble s'expliquer par la formation cl'un pont salin entre deux résidus, strictement conservés dans l'AR, le GR, le MR, le PR (R598 et I)600) pour le AR). Ia formation d'hétérodimères GR/AR [28] a cependant été rapportéc, mais avec des capacités transcriptionnelles contradictoires et sans aucune comparaison avec le rôle-clé de l'hétérodimérisation dans la régulation transcriptionnelle d'autres membres de la superfamille, tels que RXR qui interagit avec plusieurs récepteurs (RAR, TR, VDR, PPAR...).

Étude structurale du domaine de licison ì l'ADN

La majeure partie des comnaissances acquises sur le DBD des récepteurs nucléaires provient des études de GR, ER et RXR. Huit des neuf cystéines qui le composent sont engagées dans des liaisons tétraédriques de coordination avec des atomes de zinc, formant ainsi deux structures en doigt de zinc [25]. Toutes ces structures présentent une organisation tridimensionnelle similaire, reflet de la très haute conservation des séquences primaires. Nous avons ainsi pu construire par homologie un modèle du DBD de $\mathrm{AR}$ à partir des connaissances acquises sur le I)BD de GR (figure 4). Nous observons ainsi 2 monomères se liant à chacun des demi-sites, tête-à-tête. L'orientation est telle que la boîte $P\left(G_{577} S\left(C K V_{581}\right)\right.$, qui assure la spécificité de reconnaissance, est présentée au demi-site.

La triple approche clinique-modèle in silico-expérimentation in vitro nous a permis de démontrer l'importance des résidus V581 et R585* dans l'interaction avec l'ADN, et de préci-

* Corde à une lettre des acides aminés: A: Ala; $C: C, \mathrm{C}$ I): Asp; E: Glu; F: Phe: $G: G): H: H i s ; I: I l e: K$ : L.js; L.: Ieu; M: Met; N: Asn; P: Bro; (): Gln;R: Arg; S: Ser; T: Thr; V: Val; W: Trp; Y; Tyr.
I)'autres régions du récepteur participent aussi à la dimérisation. Ainsi, un réccpteur tronqué de la partie A/B présente une capacité de climérisation plus faible que la protéine entière [29], alors que la fonction majeure de dimérisation de l'ER se situc au niveau du LBD, notamment par l'intermédiaire de $\mathrm{HlO}$.

\section{Régulation de la transcription}

Chez les eucaryotes, l'activation de la transcription de gènes en réponse à des signaux extracellulaires nécessite l'assemblage de complexes multiprotéiques au niveau de leur région promotrice et la régulation de la synthèse d'ARNm par la RNA polymérase II.

Les récepteurs stéroïdiens stimulent la formation du complexe de préinitiation en augmentant sa stabilité et/ou sa vitesse de formation. Cependant, le mécanisme par lequel ils interagissent avec la machinerie transcriptionnelle est encore peu connu et pourrait impliquer des interactions directes et/ou indirectes.

\section{Interactions directes} dence in vitro mais aussi in vivo, des
McEwan et al. [30] ont mis en évi- 

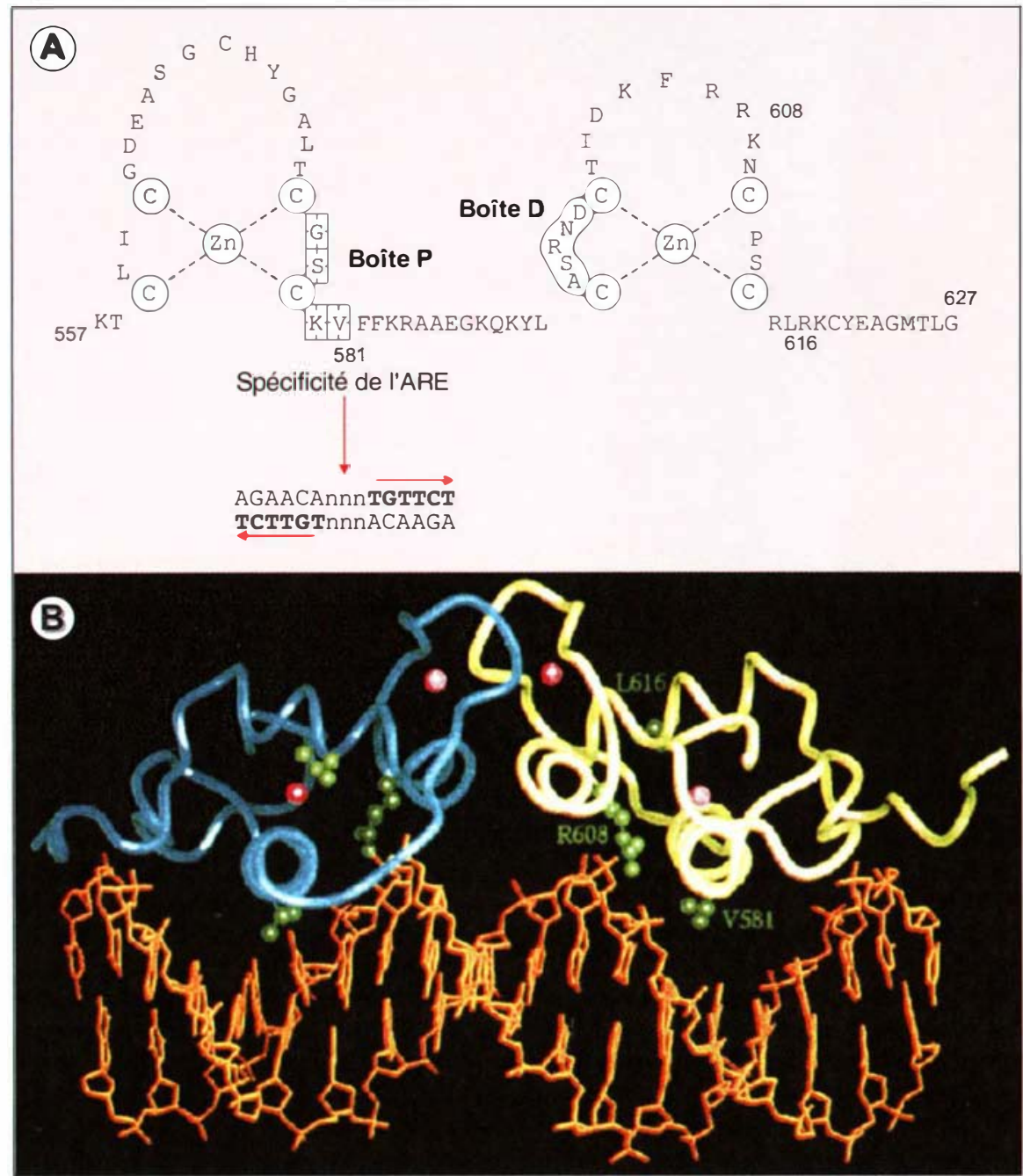

Figure 4. A. Domaine de liaison de I'ADN du récepteur des androgènes. Représentation linéaire et localisation des régions fonctionnelles, notamment de la boîte $P$, impliquée dans la reconnaissance de l'ADN, et de la boîte $D$, impliquée dans les phénomènes de dimérisation. B. Vue de l'architecture générale du domaine de liaison de I'ADN du récepteur des androgènes, modèle $3 D$ réalisé à partir des coordonnées du complexe rGR-GRE4s. En jaune figure le monomère qui interagit spécifiquement avec I'ADN, les sphères roses représentant les atomes de zinc.

interactions entre le domaine N-terminal du AR et TFIIF. I) même Ford el al. [31] ont rapporté des interactions entre le domaine Tau-l $\left(t_{1}\right)$ de (iR et TBP. I.e I)BI) de PR présente lui des interactions avec $T_{A F_{11}} 110$, alors que pour la région AF2 de ER la cible est $\mathrm{TAF}_{||} 30$ [32].

Interactions indirectes

I 'identification de la région $\mathrm{AF} 2$, associée à l'observation d’interférences transcriptionnelles réciproques cle dif-
$S R C / N C o A$

A ce jour, trois membres distincts ont été décrits, chacun présentant plusieurs variants d'épissage [33] : SRC1/NCoa-1, TIF2/GRIP-1 et p/CIP. SRC-1 stimule l'activation transcriptionnelle de plusieurs récepteurs nucléaires, dont le $A R$, en réponse à leurs ligands respectifs, même si le AR semble aroir une préférence pour TIF2 [35].

I.e domaine d'interaction SRC-récepteur nucléaire comprend quatre motifs très conservés I.XXI.I. (où X est n'importe quel acide aminé et $\mathrm{I}$. une leucine) $\left(\mathrm{m} / \mathrm{s} 1997, n^{\circ} 10\right.$, p. 1212). Des mutations du second motif affectent grandement l'interaction avec ER et RAR, alors que ce sont plutôt des altérations des motifs III et IV qui perturbent la liaison avec le AR. I e module IV présente notamment une interaction préferentielle avec le domaine d'activation transcriptionnelle AFl, localisé dans la partie N-terminale du AR. SRC:-1 pourrait clonc participer à l'interaction entre les parties amino- et carboxy-terminales du RA $[36,37]$.

L'une des explications du mécanisme d'activation par ces co-activateurs a été apportée par la découverte d'une co-activation synergique SR(:-1(.BP/p300. CBP et $\mathrm{p} / 300$ sont deux protéines apparentées, capables d'interagir avec les récepteurs nucléaires mais aussi avec divers facteurs de transcription tels CREB (cAMP response element binding protein), AP-1 (activator protein 1), STAT (signal transducer and activator of transcription). ('e dernier point leur a valu l'appellation d'intégrateurs de diverses voies de signalisation. Elles possèdent une activité histone-acétyltransférase intrinsèque (HAT) et peuvent clonc agir de concert afin de remodeler la chromatine.

On peut donc imaginer qu'un complexe multiprotéique, comprenant ('BP, divers coactivateurs et doté d'une activité HAT, puisse être recruté par des récepteurs nucléaires en réponse à la liaison de leurs ligands respectifs. I'inactivation génique de SRC-1 n'a pas révélé de phénotype particulier [38].

\section{Ara 70/liLt:1}

I a protéine ARA70 (androgen receptor activator $70 k D a)$ a été isolée sur la base de son interaction avec le I.BI) 
du récepteur des androgènes en présence de DHT [39]. Les auteurs présentent cette protéine comme ayant une interaction spécifique avec le $\mathrm{AR}$, or elle a initialement été isolée à partir de cellules de cancer thyroïdien, et dénommée RFG ou ELEl. Ces données ne semblaient pas en faveur d'une spécificité d'interaction limitée au $\mathrm{AR}$, et ont été récemment confirmées. Non seulement ARA70/ ELEl interagit avec GR et ER mais cette interaction, comme celle avec le $\mathrm{AR}$, est indépendante du ligand [40], et ne nécessite pas les domaines I.xxI.I de ARA70/ELEl et AF2-AD du AR. La protéine ARA70/EL.E1 semble donc devoir être considérée comme un co-activateur particulier.

\section{$B A G 1 L$}

BAG-1 est un régulateur de la famille des hsp70, qui agit en augmentant les échanges ADP/ATP et dont les interactions avec divers récepteurs stéroïdiens ont été décrites. Seule l'isoforme BAG-lL est co-immunoprécipitée avec le AR et sa surexpression transitoire permet d'amplifier la réponse androgénique [41]. La nature de cette interaction reste à établir, directe ou indirecte par l'intermédiaire de hsp70. Le support de cette interaction dans le AR est le domaine de liaison de l'ADN et la région charnière, différenciant ainsi clairement $\mathrm{BAG}-\mathrm{II}$, des co-activateurs de la famille pl60.

\section{ARIP3}

ARIP3 (androgen receptor interacting protein 3) est un partenaire du RA, particulièrement exprimé dans le testicule. Cette protéine a été isolée par la technique de double hybride en utilisant comme appât le domaine de liaison à l'ADN et une partie de la région charnière [42]. ARIP3 module de manière limitée l'activité transcriptionnelle du AR. La partie amino-terminale d'ARIP3 présente de fortes homologies avec Mi\%l (interagissant avec une protéine à homéodomaine Msx-2), (;BP (interagissant avec une ARN hélicase), PIAS1 et PIAS3 (inhibant respectivement la liaison à l'ADN de Statl et Stat3). Il semble s'agir d'une nouvelle famille de protéines modulant l'activité transcriptionnelle de leurs partenaires respectifs, dont la recon- naissance serait assurée par le domaine carboxy-terminal.

\section{SRA}

I .e SRA (steroid receptor coactivator) présente une fonction de coactivation impliquant le domaine AFl des récepteurs stéroïdiens. Il s'agit en fait d'un coactivateur agissant sous forme d'ARN, qui existe au sein d'un complexe ribonucléoprotéique comprenant SRC-1, recruté par les récepteurs stéroïdiens dont le AR [43], $\left(m / s\right.$ 1999, $\left.n^{\circ} 10, p .1153\right)$. L'existence de ce nouveau type de coactivateur ne va pas sans poser de nombreuses questions: comment expliquer l'activation de tous les récepteurs stéroïdiens alors que leur domaine aminoterminal est particulièrement divergent? Ce mode de collaboration transcriptionnelle dans lequel un ARN établit une plate-forme de recrutement existe-t-il dans d'autres systèmes de contrôle de transcription?

\section{Les co-répresseurs}

Plusieurs membres de la superfamille ont une activité répressive sur les fonctions de transcription, en l'absence de ligand. Deux protéines apparentées ont été isolées, NCoR, et SMRT qui sont les médiateurs de la répression de transcription exercée par RAR et TR. Récemment, le nombre de membres de la superfamille capables d'interagir avec ces co-répresseurs s'est accru et inclut notamment PR et ER en présence d'antagonistes respectifs, RU486 [44] et le tamoxifène [45]. Ces données suggèrent que les concentrations relatives de co-répresseurs et de co-activateurs jouent un rôle prépondérant dans la définition du caractère antagoniste-agoniste partiel, sujet à variation selon le type cellulaire utilisé. Un tel équilibre peut avoir des répercussions sur la physiopathologie de la résistance aux antiandrogènes lors d'un traitement du cancer de la prostate même si à l'heure actuelle l'interaction AR-corépresseur n'a pas été démontrée. On peut se demander si la localisation cytoplasmique en l'absence de ligand de AR, de même que GR et MR, ne les dispense pas de l'interaction avec un tel complexe répresseur.

Un modèle général d'action du récepteur des androgènes se dégage de l'ensemble de ces données (figure 5): la fixation du ligand par AR cytoplasmique, formant un complexe avec diverses protéines (hsp), va déclencher une série de modifications (départ des hsp, hyperphosphorylations). AR subit alors une transloca-

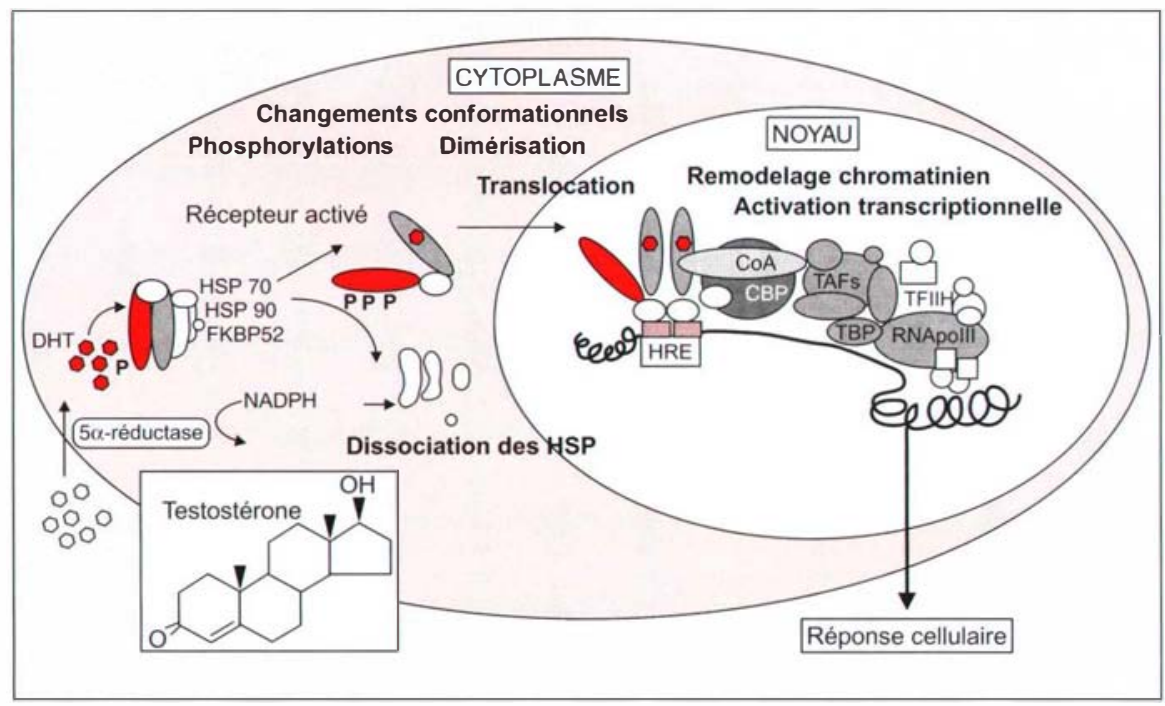

Figure 5. Mécanisme d'action du récepteur des androgènes. La fixation $d u$ ligand par le récepteur cytoplasmique, formant un complexe avec diverses protéines (hsp), va déclencher une série de modifications (départ des hsp, hyperphosphorylations). Le AR subit alors une translocation jusqu'au noyau où il va reconnaitre ses éléments de réponse hormonaux et recruter le complexe activateur de transcription. Le remodelage chromatinien subséquent permet l'assemblage de la machinerie transcriptionnelle et l'activation de la transcription. 
tion jusqu'au noyau où il va reconnaître ses éléments de réponse hormonaux et recruter le complexe activateur de transcription, constitué entre autres de protéines à activité histone-acétyl transférase. L.e remodelage de la chromatine subséquent permet l'assemblage de la machinerie transcriptionnelle et l'activation de la transcription.

\section{Modulation de l'activité transcriptionnelle}

L'activité transcriptionnelle du AR peut aussi être modulée par diverses voies de signalisation cellulaires, y compris en l'absence d'hormone. Parmi ces modulateurs de l'activité transcriptionnelle, le complexe AP1, constitué principalement des protooncogènes c-Jun et c-Fos, est particulièrement étudié. L'activité transcriptionnelle de ce complexe est subordonnée à l'action de facteurs de croissance, d'oncogènes, de cytokines, qui activent la protéine-kinase C e et conduisent à la prolifération cellulaire. I.es données de la littérature concernant ces intercommunications AR-API sont discordantes [46]. Le mécanisme d'action est également très discuté, pouvant intervenir soit par interaction directe [46] soit par compétition entre AR ct APl vis-à-vis d'un facteur limitant dans la cellule, tel que CBP [47].

Nous avons pu récemment démontrer une répression mutuelle des deux voies de signalisation du AR et d'APl [48]. En utilisant plusieurs mutants naturels et artificiels du AR, nous avons précisé les régions du réceptcur indispensables à cette interaction.

Une activation du AR indépendante des androgènes, pouvant notamment rendre compte de l'échec des traitements thérapeutique dans les cancers de la prostate, est activement recherchée. Une activation transcriptionnelle du AR a été mise en évidence en l'absence d'hormone pour plusieurs facteurs de croissance, l'IGF-1, l'EGF (epidermal growth factor) et le KGF (keratinocyte growth factor) [49] mais les mécanismes d'activation restent inconnus et les implications cliniques demeurent à définir. Très récemment, Ciraft et al. [50] ont démontré l'existence d'une nouvelle

\begin{tabular}{|l|}
\hline *ABRÉVIATIONS * \\
In silico: sur ordinateur. \\
Boîte P: séquence $G_{577} S C K V_{581}$ du \\
domaine de liaison de l'ADN impli- \\
quée dans la reconnaissance des élé- \\
ments de réponse hormonaux. \\
Boîte D: séquence $A_{596}$ SRNI ${ }_{600}$ impli- \\
quée dans les phénomènes de dimé- \\
risation. \\
ICA: syndrome d'insensibilité complète \\
aux androgènes. \\
IPA: syndrome d'insensibilité partielle \\
aux androgènes.
\end{tabular}

2/neu, un récepteur à tyrosine kinase de la famille des récepteurs à EGF. L'utilisation de lignées cellulaires issues de cancer de prostate et stablement transfectées par un gène reporter androgéno-sensible [51] devrait permettre d'approfondir les connaissances dans ce domaine.

\section{Conclusions}

I.es données relatives au mécanisme d'action cellulaire et moléculaire des androgènes dans des cellules cibles s'accumulent de façon exponentielle. La recherche actuelle se focalise sur trois axes essentiels, une approche structurale, la modulation de la signalisation du récepteur des androgènes par des facteurs de croissance et l'identification des divers partenaires du récepteur dans l'activation transcriptionnelle. Il est donc envisageable d'espérer que ces trois axes complémentaires permettent à moyen terme de définir une carte des relations structure-fonction aussi détaillée que possible. Elle favorisera une meilleure approche des maladies qui impliquent les androgènes et leur récepteur, syndromes d'insensibilité aux androgènes et cancers de la prostate, contribuant ainsi à une meilleure prise en charge de ces affections

\section{Remerciements}

A l'ensemble de l'équipe sans laquelle ce travail n'aurait pas été possible, ainsi qu'à JeanMarie Wurtz et Dino Moras (IGBMC, Strasbourg) pour leur apport déterminant lors de l'approche 3D du LBD de AR, Laurent Chiche (CBS, Montpellier) pour la modélisation du DBD.

\section{Addendum}

Depuis la rédaction de cette revue, divers cofacteurs interagissant avec RA ont été identifiés, notamment Ubc9, ANPK, FHL2 (à activité activatrice) TR4 et SHP (à activité inhibitrice).

\section{RÉFÉRENCES}

1. Sultan C, Lobaccaro JM. Génétique moléculaire des syndromes d'insensibilité aux androgènes. Med Sci 1991; 7 : 697-704.

2. Sultan C, Savage MO. Intersex states. In: G.A.B. Clinical endocrinology. London : 1998 : 796-809.

3. Quigley CA, Debellis A, Marschke KB, Elawady MK, Wilson EM, French FS. Androgen receptor defects: historical, clinical, and molecular perspectives. Endocrine Rev 1995; 16: 271-321.

4. Mangelsdorf $\mathrm{D}$, Thummel C, Beato $\mathrm{M}$, $e t$ al. The nuclear receptor superfamily: the second decade. Cell 1995; 83: 835-9.

5. Supakar PC, Song CS, Jung MH, et al. A novel regulatory element associated with age-dependent expression of the rat androgen receptor gene. J Biol Chem 1993; 268 : 26400-8.

6. LeDai J, Burnstein KL. Two androgen response elements in the androgen receptor coding region are required for cell-specific up-regulation of receptor messenger RNA. Mol Endocrinol 1996; 10 : 1582-94.

7. Nirdé $P$, Georget $V$, Térouanne $B$, Galifer RB, Belon C, Sultan C. Quantification of androgen receptor messenger RNA from genital skin fibroblasts by reverse transcription-competitive polymerase chain reaction. J Steroid Biochem Mol Biol 1998; 66: 35-43.

8. Moras D, Gronemeyer $\mathrm{H}$. The nuclear receptor ligand-binding domain: structure and function. Curr Opin Cell Biol 1998; 10 : 384-91.

9. Nolte RT, Wisely GB, Westin S, et al. Ligand binding and co-activator assembly of the peroxisome proliferator- activated receptor-gamma. Nature 1998; 395: 137-43.

10. Shiau AK, Barstad D, Loria PM, et al. The structural basis of estrogen receptor/ coactivator recognition and the antagonism of this interaction by tamoxifen. Cell 1998; 95: 927-37.

11. Wurtz JM, Bourguet W, Renaud JP, et al. A canonical structure for the ligand-binding domain of nuclear receptors. Nat Struct Biol $1996 ; 3$ : 87-94

12. Fagart J, Wurtz JM, Souque A, Hellal LC, Moras D, Rafestin OM. Antagonism in the human mineralocorticoid receptor. $E M B O J$ $1998 ; 17: 3317-25$.

13. Poujol N, Wurtz JM, Tahiri B, Lumbroso S, Nicolas JC, Moras D, Sultan C. Specific recognition of androgens by their nuclear receptor: a structure-function study. J Biol Chem 2000 (sous presse). 


\section{RÉFÉRENCES}

14. Renaud JP, Rochel N, Ruff M, et al. Crystal structure of the RAR-gamma ligand-binding domain bound to all-trans retinoic acid. Nature $1995 ; 378$ : $681-9$

15. Gottlieb B, Lehvaslaiho $H$, Beitel LK, Lumbroso R, Pinsky L, Trifiro M. The androgen receptor gene mutations database. Nucleic Acids Res 1998; 26 : 234-8.

16. Veldscholte J, Berrevoets CA, Zegers ND, Vanderkwast TH, Grootegoed JA, Mulder $\mathrm{E}$. Hormone-induced dissociation of the androgen receptor-heat-shock protein complex - use of a new monoclonal antibody to distinguish transformed from nontransformed receptors. Biochemistry 1992; 31 : 7422 30.

17. Kuil CW, Berrevoets CA, Mulder E Ligand-induced conformational alterations of the androgen receptor analyzed by limited trypsinization. Studies on the mechanism of antiandrogen action. I Biol Chem nism of antiandrogen

18. Kuiper GGJM, Brinkmann AO. Phosphotryptic peptide analysis of the human androgen receptor: detection of a hormone-induced phosphopeptide. Biochemistry mone-induced phos
$1995 ; 34: 1851-7$

19. Tremblay A, Tremblay GB, Labrie F Giguere V. Ligand-independent recruitment of SRC-1 to estrogen receptor beta through phosphorylation of activation function AF-1. Mol Cell 1999; 3: 513-9.

20. Hammer GD, Krylova I, Zhang Y, et al. Phosphorylation of the nuclear receptor SF1 modulates cofactor recruitment: integration of hormone signaling in reproduction and stress. Mol Cell 1999; 3 : 521-6.

21. Görlich D. Transport into and out of the cell nucleus. $E M B O$ J 1998; 17: 2721-7.

22. Roche PJ, Hoare SA, Parker MG. A consensus DNA-Binding site for the androgen receptor. Mol Endocrinol 1992; 6: 2229 . g.5.

23. Fabre S, Manin M, Pailhoux E, Veyssière $G$, Jean $C$. Identification of a functional androgen response element in the promoter of the gene for the androgen-regulated aldose reductase-like protein specific to the mouse vas deferent. $f$ Biol Chem 1994; 269 : 5857-64

24. Haelens A, Verrijdt G, Schoenmakers E, et al. The first exon of the human sc gene contains an androgen responsive unit and an interferon regulatory factor element. $M o l$ Cell Endocrinol 1999; 153 : 91-102.

25. Freedman LP. Anatomy of the steroid receptor zinc finger region. Endocrine Rev 1992; 13: 129-45.

26. Lobaccaro JM, Poujol N, Chiche L, Lumbroso S, Brown TR, Sultan C. Molecular modeling and in vitro investigations of the human androgen receptor DNA-binding domain: application for the study of two mutations. Mol Cell Endocrinol 1996; 116: $137-47$.
27. Poujol N, Lobaccaro J Chiche L Lumbroso S, Sultan C. Functional and structural analysis of $\mathrm{R} 607 \mathrm{Q}$ and $\mathrm{R} 608 \mathrm{~K}$ androgen receptor substitutions associated with male breast cancer. Mol Cell Endocninol 2000 (sous presse).

28. Chen S, Wang J, Yu GQ Liu W, Pearce D. Androgen and glucocorticoid recepto heterodimer formation. Proc Natl Acad So USA $1997 ; 272: 14087-92$

29. Eriksson P, Wrange O. Protein-protein contacts in the glucocorticoid receptor homodimer influence its DNA binding properties. J Biol Chem 1990; $265: 3535-42$.

30. McEwan IJ, Gustafsson J. Interaction of the human androgen receptor transactivation function with the general transcription factor TFIIF. Proc Natl Acad Sci USA 1997 $94: 8485-90$

31. Ford I, McEwan II, Wright AP, Gustafsson IA. Involvement of the transcription factor IID protein complex in gene activation by the $\mathrm{N}$-terminal transactivation domain of the glucocorticoid receptor in vitro. Mol Endocrinol 1997; 11: 1467-75.

32. Jacq X, Brou C, Lutz Y, Davidson I, Chambon P, Tora L. Human TAFII30 is present in a distinct TFIID complex and is required for transcriptional activation by the estrogen receptor. Cell 1994; 79: 107-17.

39. Torchia J, Glass $\mathrm{C}$, Rosenfeld MG. Coactivators and co-repressors in the integration of transcriptional responses. Curr Opin Cell Biol 1998; 10: 373-83.

34. McKenna NJ, Lanz RB, O'Malley BW. Nuclear receptor coregulators: cellular and molecular biology. Endocrinol Rev 1999; 20 . $321-44$

35. Ding XF, Anderson CM, Ma $\mathrm{H}$ et al. Nuclear receptor-binding sites of coactivators glucocorticoid receptor interacting protein 1 (GRIP1) and steroid receptor coactivator 1 (SRC- 1): multiple motifs with different binding specificities. Mol Endocrinol $1998 ; 12$ : 302-13.

36. Ikonen T, Palvimo II, Janne OA. Interaction between the amino- and carboxyl-terminal regions of the rat androgen receptor modulates transcriptional activity and is influenced by nuclear receptor coactivators. J Biol Chem 1997; 272: $29821-8$

37. Berrevoets CA, Doesburg P, Steketee K Trapman J, Brinkmann AO Functional interactions of the AF-2 activation domain core region of the human androgen receptor with the amino-terminal domain and with the transcriptional coactivator TIF? (transcriptional intermediary factor2). Mo Endocrinol $1998 ; 12: 1172-83$

38. Xu J, Qiu Y, DeMayo FI, Tsai SY, Tsai M], O'Malley BW. Partial hormone resistance in mice with disruption of the steroid receptor coactivator-1 (SRC-1) gene. Science $1998 ; 279: 1922-5$.

39. Yeh $\mathrm{S}$, Chang $\mathrm{C}$. Cloning and characterisation of a specific coactivator, Ara 70 , for the androgen receptor in human prostate cells. Proc Nall Acad Sci USA 1996; 93:551721.
40. Alen P, Claessens F Schoenmakers E, et al. Interaction of the putative androgen receptor-specific coactivator ARA70/ ElElalpha with maltiple steroid receptors and identification of an internally deleted ELElbeta isoform. Mol Endocrino $1999 ; 13$. 117.28 .

41. Froesch BA, Takayama S, Reed JC. BAG IL protein enhances androgen receptor function. I Biol Chem 1998; 273: 11660-6.

42. Mollanen AM, Poukka H, Karvonen U, Hakli M, Janne OA, Palvimo II. Identification of a novel RING finger protein as a coregulator in steroid receptor-mediated gene transcription. Mol Cell Biol 1998; 18: b128-39.

43. Lanz RB, McKenna NI, Onate SA, at al. A steroid receptor coactivator, SRA, functions as an RNA and is present in an SRC-1 complex. Cell 1999;97:17-27.

44. Jackson TA, Richer IK, Bain DL, Takimoto GS, Tung L, Horwitz KB. The partial agonist activity of antagonist-occupied steroid receptors is controlled by a novel hinge domain-binding coactivator L7/SPA and the corepressors N-CoR or SMRT. Mol Endocrinol 1997; 11 : 693-705.

45. Smith CL, Nawaz Z, O'Malley BW. Coactivator and corepressor regulation of the agonist/antagonist activity of the mixed antiestrogen, 4-hydroxytamoxifen. Mol Endocrinol 1997; 11:657-66.

46. Bubulva A, Wise $S G$, Shen XO, Burmeister LA, Shemshedini L. c-Jun can mediate androgen receptor-induced transactivation. I Biol Chem 1996; 271 : $24583-9$.

47. Aamisalo P, Palvimo II, Janne OA. CREB-binding protein in androgen receptor-mediated signaling. Proc Nall Acad Sci USA 1998; $95: 2122-7$.

48. Lobaccaro JM, Poujol N, Terouanne B, at al. Transcriptional interferences between normal or mutant androgen receptors and the activator protein 1: dissection of the androgen receptor functional domains. Endocrnology $1999 ; 140: 350-7$.

49. Culig Z, Hobisch A, Cronauer MV, et al. Androgen receptor activation in prostatic tumor cell lines by insulin- like growth factor-I, keratinocyte growth factor, and epidermal growth factor. Cancer Res 1994; 54 : $5474-8$.

50. Craft N, Shostak Y, Carey M, Sawyers CL. A mechanism for hormone-independent prostate cancer through modulation of androgen receptor signaling by the HER2/neu tyrosine kinase. Nat Med 1999; 5 : $280-5$. 51. Térouanne B, Tahisi B, Georget V,
Belon C, Poujol N, Avances C, Orio F Jr, Balaguer P, Sultan C. A stable prostatic bioluminescent cell line to investigate androgen and antiandrogen effects. Mol Cell Endo crinol $2000 ; 160: 3949$. 


\section{Summary}

Molecular action of androgens and structure-function relationships of androgen receptor

Androgens affect differentiation, growth and development of a wide variety of cell types in both males and females. They produce their effects upon binding to the androgen receptor (AR) which modulates the transcription of specific genes. Testosterone is the major active androgen circulating in blood, but the enzyme $5 \alpha$-reductase transforms this hormone into dihydrotestosterone, which binds to and activates the AR. The binding of hormone by cytosolic AR, complexed by several proteins (hsp), initiates a series of conformational changes resulting in the translocation of $A R$ into the nucleus where it interacts with specific DNA-sequences and recruits the transcription initiation complex. Over the past two years, significant progress has been made in the understanding of the cellular and molecular action of androgens. The molecular actors implicated in transcriptional activativation by the androgen receptor (AR) have been identified and their role in the activation process are now better defined. Modeling of the three-dimensional structure of the DNA- and ligand-binding domains of $\mathrm{AR}$ opens the way for numerous physiopathological and pharmacological applications.

\section{FONDATION FYSSEN}

\section{4, RUE DE RIVOLI - 75001 PARIS}

TÉL. : 33 (0)142975316 - FAX: 33 (0)142601795

La FONDATION FYSSEN a pour objectif général "de promouvoir sous toutes ses formes l'analyse scientifique des mécanismes logiques du comportement animal et humain ainsi que leur développement ontogénétique et phylogénétique».

\section{BOURSFS D'ÉTUDES POST-DOGTORALFS}

La FONDATION FYSSEN attribuera un certain nombre de BOURSES D'ÉTUDES POST-DOCTORALES.

Ces bourses doivent permettre la formation et le soutien de chercheurs de niveau post-doctoral travaillant dans des domaines de recherche qui répondent aux objectifs de la Fondation tels que l'éthologie, la paléontologie, l'archéologie, l'anthropologie, la psychologie, l'épistémologie, la logique et les sciences du système nerveux.

La Fondation souhaiterait soutenir plus particulièrement les recherches dans les domaines tels que:

ÉTHOLOGIE ET PSYCHOLOGIE: La nature et le développement des processus cognitifs chez l'homme et chez les animaux. Le déterminisme des comportements au cours de l'ontogenèse et leur évolution à travers la phylogenèse.

NEUROBIOLOGIE : Les études portant sur les bases neurobiologiques des processus cognitifs et de leur développement embryonnaire et post-natal ainsi que les mécanismes élémentaires qu'ils engagent.

ANTHROPOLOGIE-ETHNOLOGIE: L'étude :

a) des systèmes de représentations des environnements naturels et des cultures. Analyse des principes de construction et des mécanismes de transmission de ces systèmes en mettant en évidence leurs aspects cognitifs;

b) des systèmes techniques développés dans les diverses formes d'organisation sociale et analysés sous tous leurs aspects (savoirs, savoir-faire, mécanismes de transmission)

PALÉONTOLOGIE HUMAINE - ARCHÉOLOGIE : L'origine et l'évolution du cerveau humain et de ses productions.

Ces bourses d'un montant maximum de $120000 \mathrm{~F}$ annuel seront réservées à des chercheurs français désirant se rendre dans des laboratoires étrangers et à des chercheurs étrangers venant travailler dans des laboratoires français. Elles s'adressent aux jeunes chercheurs, moins de 35 ans, et sont normalement d'une durée maximale d'un an; elles peuvent éventuellement être renouvelées.

Les demandes de bourses doivent être établies suivant un formulaire à demander à la Fondation de Septembre à Février. Les dossiers complets doivent être adressés en 15 exemplaires au Secrétariat de la Fondation, 194, rue de Rivoli, 75001 PARIS.

Date limite impérative de réception des dossiers: le 31 mars 2000.

Seules seront prises en considération les demandes de bourses qui entrent explicitement dans les objectifs de la Fondation.

\section{PRIX INTERNATIONAL}

Un PRIX INTERNATIONAL de $200000 \mathrm{~F}$ est attribué à un chercheur qui s'est distingué par une activité de recherche fondamentale qui correspond, directement, ou indirectement à l'objectif de la Fondation et qui concerne des disciplines telles que l'éthologie, la paléontologie, l'archéologie, l'anthropologie, la psychologie l'épistémologie, la logique et les sciences du système nerveux. II a été décerné à MM. les Professeurs A. LEROI-GOURHAN (1980), W.H. THORPE (1981), V.P. MOUNTCASTLE (1982), H.C. CONKLIN (1983), R.W. BROWN (1984), P. BUSER (1985), D. PILBEAM (1986), D. PREMACK (1987), J.C. GARDIN (1988), P.S. GOLDMAN-RAKIC (1989), J. GOODY (1990), G.A. MILLER (1991), P. RAKIC (1992), L.L. CAVALLI-SFORZA (1993), L.R. GLEITMAN (1994), W.D. HAMILTON (1995), C. RENFREW (1996), M. JOUVET (1997) et A. WALKER (1998).

Discipline pour le Prix International 2000: "INTENTIONNALITÉ ET PLANIFICATION DE L'ACTION "

Les propositions de candidature doivent comporter:

- le curriculum vitæ,

- la liste des publications du candidat,

- un résumé (quatre pages maximum) du travail de recherche qui justifie l'attribution du Prix.

On ne peut se porter directement candidat. La candidature doit impérativement être présentée par une personnalité scientifique reconnue et être adressée en 15 exemplaires au Secrétariat de la Fondation, 194, rue de Rivoli, 75001 PARIS.

Date limite des propositions de candidature: le 31 OCTOBRE 2000. 